\title{
Nueva aplicación para la generación de avisos de tormentas previstas en aeropuertos
}

https://doi.org/10.31978/639-19-010-0.069

\author{
Pablo Aguayo Maldonado ${ }^{1}$ (paguayom@aemet.es) \\ María Teresa García Gómez² (magarciag@aemet.es) \\ Marcos Natanael Gómez Molina3 (mgomezm@aemet.es) \\ Carlos Jiménez Alonso ${ }^{1}$ (cjimeneza@aemet.es) \\ Cecilia Marcos Martín² (cmarcosm@aemet.es) \\ Carlos Perea Hitos ${ }^{1}$ (cpereah@aemet.es) \\ Jesús Riesco Martín' (jriescom@aemet.es) \\ Alejandro Roa Alonso² (aroaa@aemet.es) \\ José Ismael Sanambrosio Beirán ${ }^{4}$ (jsanambrosiob@aemet.es)
}

\author{
${ }^{1}$ AEMET / Delegación Territorial en Andalucía, Ceuta y Melilla \\ ${ }^{2}$ AEMET / Dirección de Producción e Infraestructuras \\ ${ }^{3}$ AEMET / Delegación Territorial en Cantabria \\ ${ }^{4}$ AEMET / Delegación Territorial en Aragón
}

\begin{abstract}
RESUMEN
En AEMET se utiliza operativamente una aplicación de emisión de boletines de rayos previstos en aeropuertos, dirigida a usuarios aeronáuticos. Con esta aplicación se prevé cada 10 minutos si en la siguiente media hora alguna célula radar con rayos asociados o una estructura de rayos no asociada a células radar, quedará parcial o totalmente dentro de un círculo de $25 \mathrm{~km}$ en torno a cualquier aeropuerto. Se ha desarrollado una nueva aplicación para la generación de estos avisos, basada únicamente en el agrupamiento de rayos. Se espera que esta nueva versión mejore los resultados de la aplicación actualmente operativa, ya que, por un lado optimiza el momento de lanzamiento de la misma al no depender de la disponibilidad de imágenes radar, y por otro lado, utiliza un procedimiento de seguimiento y extrapolación de los grupos de rayos más eficaz, a la vez que la posición relativa de los grupos de rayos y los círculos de influencia de los aeropuertos se analiza por medio de un método basado en formas geométricas. A lo largo de la primaveraverano del año 2018 se va a proceder a la verificación de los resultados de esta nueva metodología.
\end{abstract}

PALABRAS CLAVE: predicción inmediata; nowcasting; descargas eléctricas; teledetección; aplicaciones aeronáuticas.

\section{INTRODUCCIÓN}

La aplicación de emisión de boletines de rayos previstos en aeropuertos (SANAMBrosio, 2010), dirigida a usuarios aeronáuticos se ejecuta cada 10 minutos. Se trata de prever a partir de los rayos observados en los 10 minutos anteriores, y haciendo una extrapolación lineal (por desplazamiento de células radar asociadas a grupos de rayos o simplemente por el viento medio de grupos de rayos no asociados a estructuras radar), si en la siguiente media hora alguna célula radar con rayos asociados o una estructura de rayos sin asociación a células radar, va a quedar parcial o totalmente dentro de un círculo de $25 \mathrm{~km}$ en torno a cualquier aeropuerto. 
Se hacen tres extrapolaciones a 10 minutos, cada una a partir del centroide y figura geométrica representativa de la estructura convectiva (con rayos asociados) o de un grupo de rayos. Se supone que en la extrapolación se conserva el tamaño y forma de las estructuras (radar y rayos) y no hay variación en el número de rayos (ni de células radar). Si a partir de la extrapolación alguno de estos objetos se sitúa en los siguientes 30 minutos, parcial o totalmente, dentro de la zona de influencia del aeropuerto $(25 \mathrm{~km})$, se emite un aviso, salvo que ya se hubiera emitido un aviso observado previamente.

Se ha desarrollado una nueva aplicación (YRAYOAENA2018) para la generación de estos avisos, basada únicamente en el agrupamiento de rayos. En este documento se explica brevemente cuál es el procedimiento que se ha adoptado.

\section{PRINCIPALES FUENTES DE ERROR}

El análisis subjetivo del comportamiento de la aplicación actualmente operativa permite considerar que los principales errores que con ella se pueden cometer se deben a los siguientes factores:

- Aparición de nuevos rayos (nuevas células o rayos que aparecen, división y fusión de células, etc.), y desaparición de rayos por decaimiento de la actividad. Esta limitación se mantiene en esta nueva versión.

- Para la trayectoria se considera un movimiento rectilíneo uniforme. Pero puede haber cambios, tanto en la dirección como en la velocidad.

- Los objetos (radar y grupos de rayos) se consideran circulares y no cambian en la extrapolación, ni en tamaño ni en cantidad de rayos. Para la generación de avisos se considera tan solo la distancia entre el aeropuerto y el centroide de la célula radar o del grupo de rayos.

- El criterio de asociación de rayos a estructuras radar es una aproximación. A partir de ahora ya no se tienen en cuenta las estructuras radar y se va a trabajar solo con los datos de rayos.

- Por convenio los rayos no asignados a estructuras convectivas radar conforman un grupo siempre que haya más de dos rayos próximos entre sí (distancia menor de 4 km entre ellos).

- Aproximación del vector desplazamiento en los casos en que se aplica, al tomar el viento medio entre 500 y $1000 \mathrm{hPa}$.

- Problemas con el retraso o no disponibilidad de datos radar, rayos o modelo:

1. Si no hay datos de composición nacional de PPI de reflectividad, se detiene hasta el próximo lanzamiento 10 minutos después. Como máximo se acepta una imagen de 30 minutos antes de la hora nominal de ejecución, (esto parece mucho, y con bastante frecuencia se están utilizando operativamente datos de 20 minutos antes).

2. No hay control acerca de si algún radar está caído y la composición nacional no es óptima. Además en esta composición nacional no entran las islas Canarias.

3. Tampoco se controla si no se han ingestado bien los rayos en McIdas (además no se consideran las descargas sino solo los rayos). Este problema se mantiene, ya que este control se tiene que efectuar desde fuera de la aplicación.

4. También puede no estar disponible el modelo numérico, en cuyo caso puede fallar la extrapolación o no se hace lo mejor posible. Esta limitación puede seguir existiendo. 


\section{MEJORAS EN LA NUEVA APLICACIÓN}

Con las modificaciones que se introducen en la nueva aplicación se va a conseguir, por un lado, optimizar la hora de generación de los avisos de rayos, ya que no depende de la disponibilidad de la imagen radar, que tienen un retraso de casi 10 minutos. Al usar solo datos de rayos, la disponibilidad es casi inmediata. Se puede lanzar la aplicación en los minutos $1(01,11,21,31,41$ y 51 de cada hora) para analizar los rayos de los 10 minutos anteriores (rayos entre los minutos 00 y 10 cuando la lanzamos en el minuto 11, por ejemplo), ganando así 9 minutos en la elaboración y emisión de los posibles avisos. Con esta nueva aplicación se generarían también los avisos para los aeropuertos de las islas Canarias.

También se va a mantener la misma numeración de los grupos de rayos durante su ciclo de vida. Por otro lado se pasan a usar modelos de mayor resolución: el HRES-IFS del ECMWF, de 16 km de resolución y el HIRLAM de alta resolución (HNR). Se seguirán usando los datos de modelo pero de una manera más eficaz, por medio del procedimiento de tracking y extrapolación que utiliza la aplicación YRADAR3D (SANAMBROSIO, 2011). En base a este cambio en la nueva aplicación las trayectorias del tracking y extrapolación de los grupos de rayos deberían mejorar, ya que el procedimiento que se usa en el YRADAR3D es más consistente y da mejores resultados que el que se usaba hasta ahora.

Para generar los avisos en esta nueva versión se va a considerar por un lado la distancia entre el aeropuerto y el centroide del grupo de rayos, y además, la posición del aeropuerto en relación con un rectángulo que inscribe a cada grupo de rayos. Este proceso se detalla más adelante.

Se va a poder disponer de la representación gráfica de la evolución de los grupos de rayos. Así, sobre la imagen de satélite (IR10.8 o HRVIS) se podrían dibujar los objetos de rayos, representados por sus rectángulos, y sus extrapolaciones cada diez minutos hasta media hora, así como la posición de aquellos aeropuertos que van a ser afectados por grupos de rayos, con el círculo establecido para generar el aviso $(25 \mathrm{~km})$. En la imagen aparecería también un listado de los grupos de rayos identificados con sus características.

En esta nueva aplicación los avisos se generan siempre, independientemente de que se hayan registrado rayos observados en los 10 minutos previos cerca de los aeropuertos. De momento no se emiten boletines de cese de actividad cuando ha dejado de haber tormentas que afectan a algún aeropuerto. Esto es algo que se podría considerar. Tampoco se incluye en los mensajes información sobre el número de rayos asociados inicialmente a las «tormentas previstas» que se espera afecten al aeropuerto o sobre si la actividad tormentosa estaba en el momento de emitir el boletín, aumentando o disminuyendo. A petición del usuario se podrían incluir otros puntos de interés aparte de los aeropuertos. Los avisos que se generan son para todo el periodo entre 0 y 30 minutos. Se podría plantear hacer los avisos por separado para cada intervalo, hasta 10 minutos, entre 10 y 20 , y entre 20 y 30 minutos.

Sería interesante mantener un archivo intensivo de producto gráfico de la aplicación, así como de ficheros de texto con las características de los grupos de rayos identificados y de los avisos. Esto permitiría en el futuro validar y llevar a cabo verificaciones sobre el comportamiento de esta aplicación.

\section{CARACTERÍSTICAS DE LOS GRUPOS DE RAYOS}

Se considera que dos o más rayos forman un grupo cuando la distancia entre ellos es inferior o igual a $4 \mathrm{~km}$. De esta manera un rayo pertenece a un grupo si está a una distancia inferior o igual a $4 \mathrm{~km}$ de alguno de los rayos que forman ese grupo. Se calcula la posición del centroide de cada grupo de rayos por medio de la latitud media y de la longitud media de todos los rayos del grupo. 


$$
L A T C=\frac{1}{N} \sum_{1}^{N} L A T_{i} \quad L O N C=\frac{1}{N} \sum_{1}^{N} L O N_{i}
$$

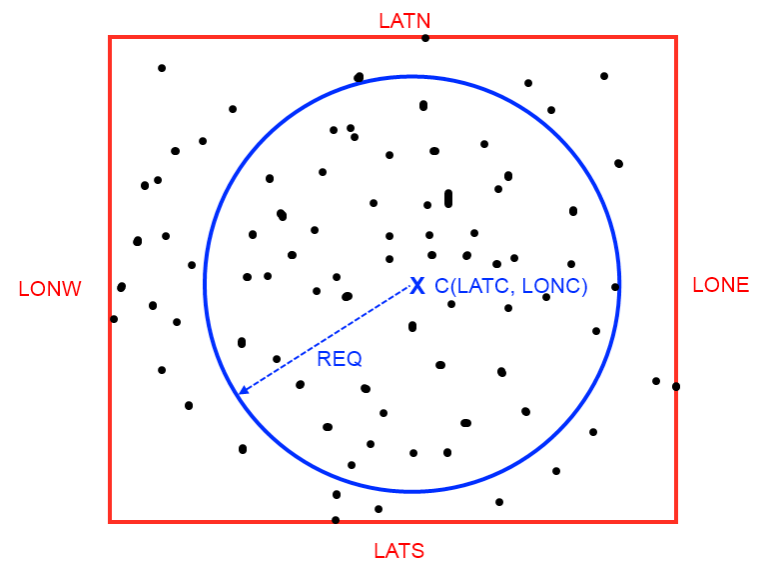

Figura 1.

Ejemplo de grupo de rayos y características que se extraen del mismo. Se aprecian los rayos individuales (puntos negros), el círculo equivalente, en azul, con la posición del centroide, $\mathrm{X}$ azul (C, identificado por su LATC y LONC), con su radio equivalente (REQ).

Se representa también, en rojo, el rectángulo que inscribe al grupo, identificado por las coordenadas LATN, LATS, LONE y LONW.

Se calcula también el radio equivalente (REQ) como la distancia media de las distancias de los rayos al centroide del grupo, y la densidad de rayos del grupo (DENR) como el número de rayos dividido por el área del círculo equivalente $\left(\pi * R E Q^{2}\right)$. Se define también un rectángulo que inscribe al grupo de rayos por medio de LATN, LATS, LONE y LONW que son respectivamente la latitud del rayo situado más al N del grupo, la del situado más al $\mathrm{S}$, la longitud del rayo situado más al $\mathrm{E}$ y la del situado más al $\mathrm{W}$ del grupo de rayos.

\section{PROCESO DE GENERACION DE AVISOS DE RAYOS}

Para la generación de avisos se va a partir de la extrapolación de los centroides de los grupos de rayos identificados, considerando las posiciones que van a tener cada 10 minutos hasta un máximo de 30, es decir se consideran solo 3 posiciones futuras. Esta extrapolación de los centroides se aplica también a los rectángulos definidos para cada grupo. En la figura 2 se ven las posiciones reales y extrapoladas de dos grupos de rayos en relación con la posición de un aeropuerto. El proceso de generación de posibles avisos se desarrolla en tres fases sucesivas, tal y como sigue:

1. Para cada intervalo de predicción (10,20 y 30 minutos) se calcula la distancia entre cada aeropuerto y la posición extrapolada de los centroides de cada grupo de rayos. Si esta distancia es inferior a la distancia establecida como radio de influencia del aeropuerto $(25 \mathrm{~km})$ se generará un aviso por tormentas previstas en dicho aeropuerto, si no se cumple esta condición, se pasará a la fase siguiente. En la figura 2 se aprecia la posición de un aeropuerto con su radio de influencia (Ra), la posición de dos grupos de rayos (G-1 y G-2) con la extrapolación y posición prevista de los centroides a 10, 20 y 30 minutos. El grupo G-1 no generará aviso en esta primera fase a partir de la primera posición, ya que la distancia d1 es mayor que Ra, pero sí lo generará en las otras dos posiciones, ya que d2 y d3 son menores que Ra. El grupo G-2 no dará lugar a avisos en esta fase.

Figura 2.

Posición actual y posiciones extrapoladas hasta 30 minutos de los centroides de dos grupos de rayos (G-1 y G-2) respecto a un aeropuerto y su radio de influencia (Ra) para generar avisos. El grupo G-1 dará lugar a avisos en sus posiciones de $20 \mathrm{y}$ 30 minutos, ya que las distancias $\mathrm{d} 2 \mathrm{y} \mathrm{d} 3$ son menores que el radio de influencia Ra.

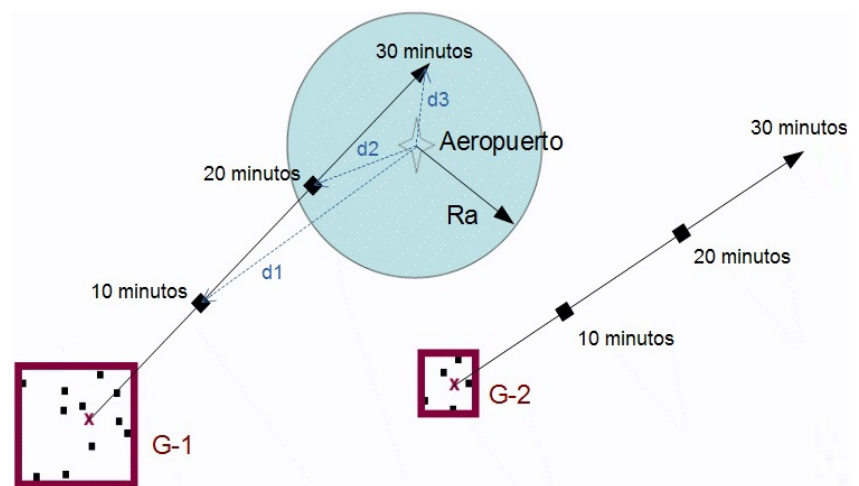


2. En cada intervalo de predicción, si no se cumple la condición anterior, se pasan a analizar las posiciones previstas del rectángulo que inscribe a cada grupo de rayos con relación al círculo de influencia de cada aeropuerto, definido por el radio Ra. En un primer caso se consideran los rectángulos pequeños, es decir, aquellos que tienen la base $(B)$ o la altura $(\mathrm{H})$ inferiores a $30 \mathrm{~km}$; si el rectángulo cumple esta condición (figura 3) se analiza si el punto más próximo del círculo de influencia del aeropuerto al centroide (Pa) se sitúa dentro del rectángulo extrapolado del grupo de rayos considerado (G-1).

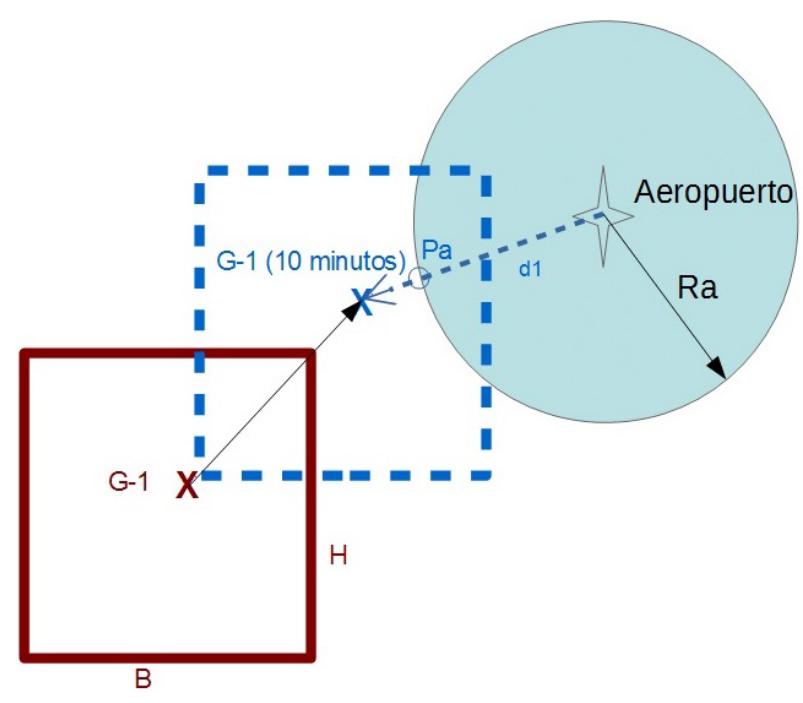

Figura 3.

Posición actual y posición extrapolada a 10 minutos del centroide del grupos de rayos G-1 y de su rectángulo (rectángulo pequeño). Aunque la distancia (d1) entre el aeropuerto y el centroide extrapolado es superior al radio de influencia $(\mathrm{Ra})$ se generaría un aviso ya que el punto más próximo $(\mathrm{Pa})$ del círculo de influencia a dicho centroide queda dentro del rectángulo extrapolado (rectángulo en línea azul discontinua). Se haría un análisis similar con la posición extrapolada a 20 y a 30 minutos.

3. La tercera fase del análisis sería la que se efectúa cuando el rectángulo que inscribe al grupo de rayos es grande, es decir, cuando tanto la base como la altura del mismo tienen más de $30 \mathrm{~km}$. En este caso se dividen la base (B) y la altura $(\mathrm{H})$ del rectángulo en 3 segmentos iguales de manera que el rectángulo queda dividido en $3 \times 3$ parcelas de idéntico tamaño (figura 4). A continuación se analiza, como en el caso anterior, si el punto más próximo del círculo de influencia del aeropuerto a la posición extrapolada del centroide del grupo de rayos (Pa) se sitúa dentro del rectángulo extrapolado del grupo considerado. Si es así, se identifica en cuál de las 9 parcelas se sitúa ese punto y se efectúa un recuento de los rayos que hay dentro de esa parcela, recalculando la densidad de rayos (DENR) para esa parcela del rectángulo. Se emitirá un aviso para ese aeropuerto cuando en la parcela considerada haya más de 2 rayos y además la densidad de rayos (DENR) recalculada para esa parcela del rectángulo sea superior a 0,1591 rayos $/ 10 \mathrm{~min} / \mathrm{km}^{2}$.

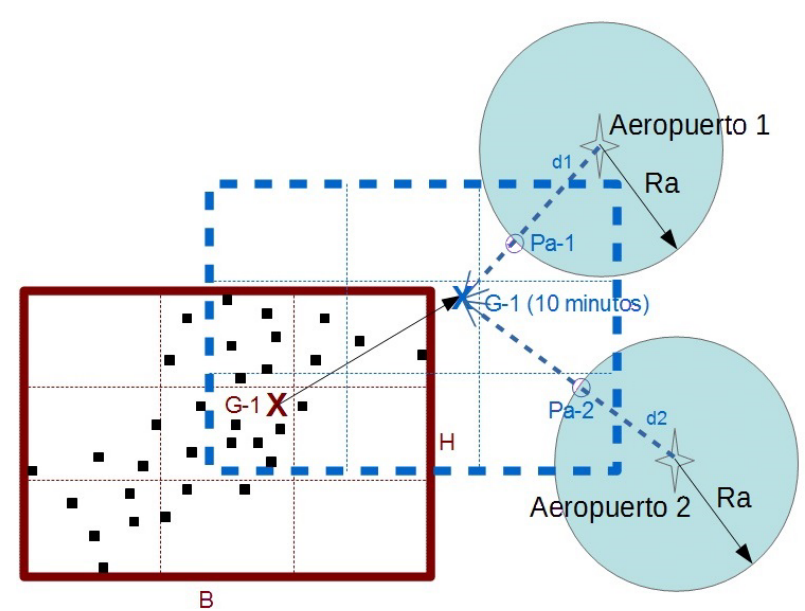

Figura 4.

Posición actual y posición extrapolada a 10 minutos del centroide del grupos de rayos G-1 y de su rectángulo. En este caso se trata de un rectángulo grande ya que tanto la base (B) como la altura $(\mathrm{H})$ tienen más de $30 \mathrm{~km}$. $\mathrm{Al}$ ser así, el rectángulo se divide en $3 \times 3$ parcelas (líneas finas discontinuas). Las distancias $\mathrm{d} 1 \mathrm{y} \mathrm{d} 2$ entre $\operatorname{los}$ dos aeropuertos y el centroide extrapolado (G-1 10 minutos) es superior al radio de influencia $(\mathrm{Ra})$, de manera que se pasaría a analizar la posición de los puntos más próximos del círculo de influencia de los aeropuertos al centroide extrapolado (puntos Pa-1 y Pa-2). En el caso del Aeropuerto 1 vemos que el punto Pa-1 de su círculo de influencia se sitúa dentro de la parcela superior izquierda

del rectángulo extrapolado (rectángulo en línea azul discontinua). En esta parcela hay cierto número de rayos (puntos negros dentro del rectángulo original de color granate) y que dan una densidad de rayos (DENR) suficientemente alta para que se genere un aviso para este Aeropuerto 1. En el caso del Aeropuerto 2 vemos que el punto Pa-2 se sitúa en una parcela que no contiene rayos en su posición original, por lo que no habría aviso para el Aeropuerto 2. 


\section{CONCLUSIONES}

Dentro del Grupo de Trabajo Radar se ha desarrollado una nueva versión de la aplicación para generar avisos de tormentas previstas en Aeropuertos. Se espera que esta nueva versión mejore los resultados de la aplicación actualmente operativa, ya que por un lado optimiza el momento de lanzamiento de la misma al no depender de la disponibilidad de imágenes radar, adelantando la generación de los posibles avisos en casi 10 minutos. Por otro lado utiliza un procedimiento de seguimiento y extrapolación de los grupos de rayos identificados a priori más eficaz, a la vez que el análisis de la posición relativa de los grupos de rayos y los círculos de influencia de los aeropuertos se lleva a cabo usando un método más complejo, basado en los rectángulos que inscriben los grupos de rayos. A lo largo de la campaña de primavera-verano de 2018 se va a proceder a la verificación de los resultados de esta nueva metodología comparándolo con los que se obtienen con la versión anterior.

\section{REFERENCIAS}

SANAmbrosio Beirán, I., 2010. Especificaciones técnicas de la aplicación de avisos de tormentas previstas para aeródromos. AEMET, MPO-ESP-0312.

Sanambrosio Beirán, I., 2010. Aplicación para generar avisos de tormentas previstas para aeródromos. AEMET, MPO-ESP-0313.

SAnAmbrosio BeIRÁn, I., 2011. Aplicación para la caracterización objetiva de estructuras y células convectivas mediante radar regional. Área de Técnicas y Aplicaciones de Predicción / Delegación Territorial en Aragón. AEMET. 